\title{
Negative regulatory responses to metabolically triggered inflammation impair renal epithelial immunity in diabetes mellitus
}

\author{
Nelson K. F. Chen • Tsung Wen Chong • \\ Hwai-Liang Loh • Kiat Hon Lim • Valerie H. L. Gan • \\ Marian Wang • Oi Lian Kon
}

Received: 6 April 2012 /Revised: 5 October 2012 /Accepted: 8 October 2012 /Published online: 14 November 2012

(C) The Author(s) 2012. This article is published with open access at Springerlink.com

\begin{abstract}
Diabetes mellitus is characterized by chronic inflammation and increased risk of infections, particularly of tissues exposed to the external environment. However, the causal molecular mechanisms that affect immune cells and their functions in diabetes are unclear. Here we show, by transcript and protein analyses, signatures of glucose-induced tissue damage, chronic inflammation, oxidative stress, and dysregulated expression of multiple inflammation- and immunity-related molecules in diabetic kidneys compared with non-diabetic controls. Abnormal signaling involving
\end{abstract}

Electronic supplementary material The online version of this article (doi:10.1007/s00109-012-0969-x) contains supplementary material, which is available to authorized users.

N. K. F. Chen $(\bowtie) \cdot$ O. L. Kon $(\bowtie)$

Division of Medical Sciences, Humphrey Oei Institute of Cancer Research, National Cancer Centre,

Singapore 169610, Singapore

e-mail: nelsonkfchen@gmail.com

e-mail: dmskol@nccs.com.sg

T. W. Chong • V. H. L. Gan

Urology Centre, Singapore General Hospital,

Singapore 169608, Singapore

H.-L. Loh $\cdot$ K. H. Lim $\cdot$ M. Wang

Pathology Department, Singapore General Hospital,

Singapore 169608, Singapore

\section{O. L. Kon}

Department of Biochemistry, Yong Loo Lin School of Medicine, National University of Singapore,

8 Medical Drive,

Singapore 117597, Singapore

Present Address:

M. Wang

Centre for Forensic Medicine, Health Sciences Authority,

Singapore 169078, Singapore cytokines, G-protein coupled receptors, protein kinase C isoforms, mitogen-activated protein kinases, nuclear factor- $\mathrm{kB}$ (NFKB), and Toll-like receptors (TLR) were evident. These were accompanied by overexpression of negative regulators of NFKB, TLR, and other proinflammatory pathways, e.g., A20, SOCS1, IRAK-M, IkB $\alpha$, Triad3A, Tollip, SIGIRR, and ST2L. Anti-inflammatory and immunomodulatory molecules, e.g., IL-10, IL-4, and TSLP that favor $\mathrm{T}_{\mathrm{H}} 2$ responses were strongly induced. These molecular indicators of immune dysfunction led us to detect the cryptic presence of bacteria and human cytomegalovirus in more than one third of kidneys of diabetic subjects but none in non-diabetic kidneys. Similar signaling abnormalities could be induced in primary human renal tubular epithelial (but not mesangial) cell cultures exposed to high glucose, proinflammatory cytokines and methylglyoxal, and were reversed by combined pharmacological treatment with an antioxidant and a PKC inhibitor. Our results suggest that diabetes impairs epithelial immunity as a consequence of chronic and inappropriate activation of counter-regulatory immune responses, which are otherwise physiological protective mechanisms against inflammation. The immune abnormalities and cryptic renal infections described here may contribute to progression of diabetic nephropathy.

Keywords Diabetes mellitus · Inflammation · Immune homeostasis $\cdot$ Renal epithelium $\cdot$ Bacterial infection . Cytomegalovirus

\section{Introduction}

Many studies have shown infection to be a major cause of death among diabetics [1-5]. A prospective 15-year study revealed a 41 -fold higher risk of death from infections 
compared with the referent population [5]. Host-specific factors have been implicated as contributing to abnormalities of innate and adaptive immunity [6-9]. Abnormalities of innate immunity mediated by leukocytes, macrophages, natural killer, and dendritic cells have been observed in diabetes [7-9]. Disordered cell-mediated immunity in diabetes is reflected in reduced leukocyte adherence, chemotaxis and phagocytosis, impaired oxidative burst and intracellular bactericidal activities, and diminished production of antimicrobial cytokines [7, 10-13]. Diabetes also impairs adaptive immunity via the cell-mediated arm [14, 15] and humoral immunity [7]. Despite evidence of diverse alterations of immune function in vitro, their relevance to the risk of infections among diabetic patients in vivo is controversial [16-18]. Nonetheless, indubitable evidence correlates good glycemic control with improved immune function and lower infection rates [1, 14, 19].

Here, we describe mechanisms that impair epithelial immunity in kidneys in human diabetes. We hypothesized that cross-talk between immune cells and resident tissue cells could be key in maintaining tissue immune homeostasis and mounting appropriate immune responses. Epithelial cells can detect microbial infections and are indispensable in "coaching" the behavior of immune cells [20, 21].

We chose kidney as a model tissue because urinary tract infection is common among diabetics and diabetic end-stage renal disease remains a major clinical challenge. We analyzed histologically normal, fresh human kidney tissues procured from non-diabetic and diabetic subjects who had clinically normal renal function. The use of non-nephropathic kidney tissues allowed us to document molecular perturbations that precede clinical complications of chronic diabetes, unconfounded by renal parenchymal disease. As diabetesassociated target tissue injury occurs before loss of clinical function [22], documenting pre-clinical molecular dysregulations may enable more effective approaches to prevent and treat diabetes-induced renal dysfunction.

Our results suggest that diabetes and hyperglycemia per se impair renal epithelial immunity as a consequence of inappropriately activated negative counter-regulatory responses, which are physiological protective mechanisms against chronic inflammation. The immune abnormalities and cryptic bacterial and viral infections we discovered in the kidneys of diabetic subjects may predispose to diabetic renal disease.

\section{Materials and methods}

Human kidney tissues

DNA, RNA, and protein were extracted from tumor-distant tissues of kidneys surgically removed for renal carcinoma.
All patients received no anticancer treatment before nephrectomy. Bacteria and human CMV were detected in DNA from fresh and formalin-fixed paraffin-embedded (FFPE) kidney tissues. Table S1 in the Electronic supplementary material (ESM) summarizes the clinical profiles of all patients in this study which was approved by the SingHealth Institutional Review Board.

DNA, RNA, and protein isolation from human kidney

Kidney tissues, flash frozen in liquid nitrogen, were verified to be cancer free and to have no diagnostic features specific for diabetic nephropathy by histological examination. None of the patients had clinical evidence of urinary infection or significant albuminuria. Frozen tissues were diced in AllProtect tissue reagent (Qiagen), immersed in tissue lysis buffer for DNA, RNA, or protein isolation and disrupted in Tissuelyser LT (Qiagen). DNA, RNA, or proteins were purified from tissue lysates using the appropriate kits and protocols (Qiagen). Membrane proteins were isolated with Q proteome cell compartment kit (Qiagen). On-column digestion of DNA was performed during RNA isolation using RNase-free DNase (Qiagen).

Antibodies and reagents

Suppliers of antibodies, reagents, and kits were: antibodies against IRAK isoforms, MyD88, RIG-1, A20, SOCS1, NIK and $\mathrm{I} \kappa \mathrm{B} \alpha, \mathrm{NF} \kappa \mathrm{B}$ p65, p38 MAPK, JNK, Akt, and their phosphorylated proteins (Cell Signaling Technology); antibodies against actin, Triad3A, TRAF6, protein kinase $\mathrm{C}$ (PKC)- $\beta$ II, Sod2, Nox4, and goat IgG-HRP (Santa Cruz Biotechnology); antibodies against TLR4 and phosphorylated PKC- $\beta$ II (Abcam); clinical grade CellGro ${ }^{\circledR}$ GMP TNF$\alpha$, IL-6, and IL-1 $\beta$ (CellGenix GmbH); methylglyoxal, lipopolysaccharide (LPS) from Escherichia coli 055:B5, lipoteichoic acid from Staphylococcus aureus, diphenyleneiodonium chloride (DPI), and Gö6983 (Sigma-Aldrich); poly(I:C) (Pharmacia Biotech); iScript ${ }^{\mathrm{TM}}$ cDNA synthesis kit (Bio-Rad); protease inhibitor cocktail (Roche), and western blot reagents (Amersham ECL ${ }^{\mathrm{TM}}$ blocking agent, Advance western blotting detection kit, and mouse- and rabbit IgG-HRP antibodies) were from GE Healthcare.

\section{Cell culture}

Clonetics ${ }^{\circledR}$ human primary renal tubular epithelial cells (RPTEC) and mesangial cells (NHMC), purchased from Lonza BioSciences, were cultured in REGM ${ }^{\mathrm{TM}}$ Renal Epithelial Cell Growth Medium and NHMC in MsGMTM Mesangial Cell Growth Medium, respectively. Cells were subcultured with ReagentPack ${ }^{\mathrm{TM}}$ Subculture Reagents. For all experiments, $2.5 \times 10^{4} \mathrm{RPTEC} /$ well or $3.5 \times 10^{4} \mathrm{NHMC} /$ 
well were seeded in a six-well plate at $37{ }^{\circ} \mathrm{C}, 5 \% \mathrm{CO}_{2}$ and cultured for 3 days before experiments commenced. Cells having the same experimental treatment were lysed directly in situ and pooled for total RNA and protein isolation. Endotoxin levels of culture media were assayed with an endotoxin detection kit (Lonza Biosciences).

\section{Western blot analysis}

Protein concentrations of fresh frozen kidney tissue lysates ( $n=8$ each for diabetic and non-diabetic samples) and primary renal cell lysates were quantitated using the bicinchoninic acid method (Thermo Scientific). Lysates were resolved on SDS-PAGE and transferred onto PVDF membranes using Trans-blot Semidry (Bio-Rad). Immunoblotting was performed using individual primary antibodies, goat-, mouse- or rabbit IgG-HRP secondary antibodies, proprietary blocking, and detection reagents stated above.

\section{Quantitative RT-PCR}

Transcript levels of 90 genes in fresh frozen human kidney tissues and primary renal cell samples were assayed with custom 96-gene $\mathrm{RT}^{2}$ Profiler ${ }^{\mathrm{TM}}$ PCR arrays (SABiosciences, Qiagen). Six diabetic kidneys positive for both bacteria and CMV were compared with six non-diabetic kidneys that were negative for both. Briefly, first-strand cDNA was generated with $\mathrm{RT}^{2}$ First Strand Kit. Quantitative PCR was performed using $\mathrm{RT}^{2}$ quantitative polymerase chain reaction (qPCR) master mix and proprietary primer pairs for each of the 90 genes (Bio-Rad CX96 thermal cycler). The transcript level of each gene was normalized with reference to $G A P D H, A C T B$, and RPL13A. Criteria for efficiency of reverse transcription and PCR, and genomic DNA contamination met the standards specified by the supplier.

\section{Quantitation of cytokines}

Levels of 20 cytokines in fresh frozen kidney tissue lysates ( $n=9$ each for diabetic and non-diabetic samples) and primary renal cell lysates were quantitated by a sandwichELISA method using a custom Quantibody ${ }^{\circledR}$ Human Cytokine Array (RayBiotech, Inc.). Fluorescence signals acquired with Axon GenePix (Molecular Devices) were analyzed with RayBio ${ }^{\circledR}$ Q Analyzer (RayBiotech, Inc.)

\section{Detection of microbial DNA in kidneys}

FFPE kidney tissues of patients of known diabetes status were sectioned for DNA isolation using QIAamp DNA FFPE tissue kit (Qiagen). Sections from the surface of tissue blocks were not used. Quantitative PCR using GoTaq ${ }^{\mathbb{R}}$ qPCR master mix (Promega) and primer pairs that amplify a $1.3-\mathrm{kb}$ DNA sequence specific to bacteria [23] was performed on $50 \mathrm{ng}$ DNA isolated from FFPE or fresh frozen kidney tissues. The amount of bacterial DNA in the sample was determined from a standard curve generated from unrelated human DNA spiked with known amounts of bacterial DNA isolated from Lactobacillus sp. and E. coli. CMV DNA was detected using Artus ${ }^{\circledR}$ CMV RG PCR kit (Qiagen). Table S1 in the ESM lists the source of tissues for microbial DNA analysis.

Data analysis

All data were expressed as mean \pm SEM. Statistical analyses (GraphPad Prism ${ }^{\circledR}$ ) between groups were performed with parametric, unpaired Student's $t$ test. Welch's correction was included if the variance was unequal. Linear correlation was performed to establish association.

All genes and proteins studied are listed in functional groups in Table S2 in the ESM.

\section{Results}

Kidney tissues express molecular signatures of diabetes

Fresh frozen tumor-free kidney tissues were obtained from nine type 2 diabetic and nine non-diabetic subjects of comparable age, body mass index, and gender distribution (Table S1 in the ESM). The diabetes status of each patient was identified by the most recent glycated hemoglobin $\left(\mathrm{HbA}_{1 \mathrm{c}}\right)$ level and/or a record of current treatment for diabetes. All patients had unimpaired renal function (serum creatinine, $\leq 110 \mu \mathrm{M}$ (males) or $\leq 85 \mu \mathrm{M}$ (females)).

By quantitative RT-PCR, immunoblotting, and antibody arrays, diabetic renal tissues showed increased activity of the polyol and hexosamine pathways, PKC signaling, and increased expression of $A G E R$ compared with non-diabetic controls (Fig. 1) Other known abnormalities detected were increased expression of NFAT5, EGR1, SPP1, INSR, and $A G T R 1$. Increased transcription of extracellular matrix protein genes, BGN, FN1, COL1A1, and THBS1 coupled with decreased expression of E-cadherin in diabetic kidneys was consistent with epithelial-mesenchymal transition which has been implicated in the pathogenesis of diabetic nephropathy [24].

These data supported the use of tumor-free kidney tissues for studying tissue effects of diabetes unconfounded by renal parenchymal disease. Transcript levels of $A K R 1 B 1$, SORD, AGER, TGFB1, PAI1, PRKCB, EDN1, VEGFA, $B G N, F N 1, C O L 1 A 1, T H B S 1, E G R 1$, and NFAT5, all implicated in diabetes complications, correlated significantly with $\mathrm{HbA}_{1 \mathrm{c}}$ levels of diabetic subjects (Fig. S1 in the ESM), confirming molecular effects of hyperglycemia on renal tissue even before functional impairment. 

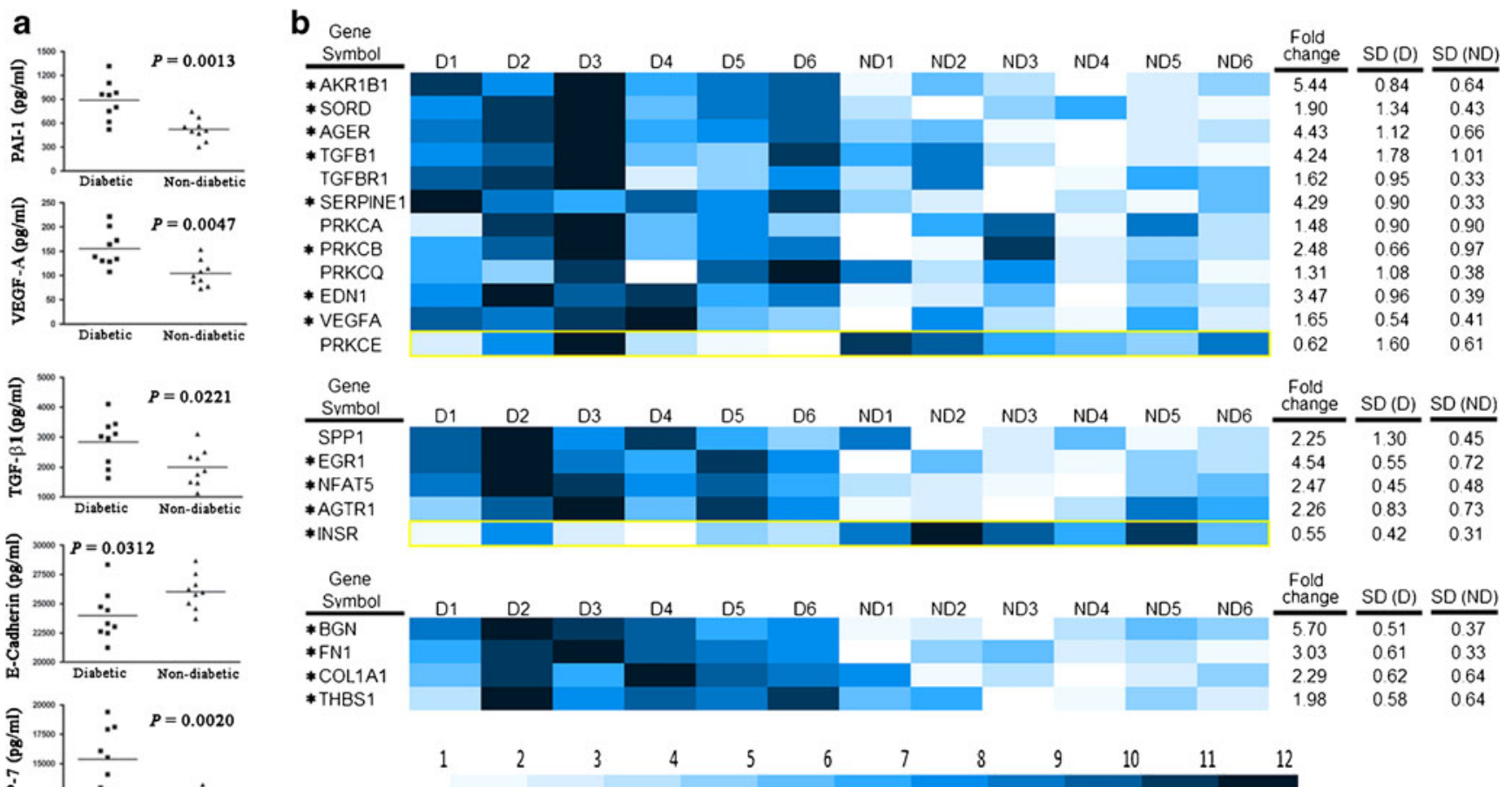

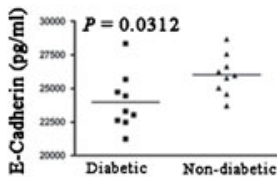

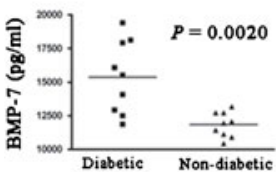

Rank

(Transcript levels)

Fig. 1 Tumor-free tissues from nephrectomized diabetic kidneys show molecular features of diabetes mellitus. a Protein lysates of histopathologically qualified tumor-free renal cortical tissues from diabetic and non-diabetic patients were assayed for the proteins indicated using a multiplex ELISA-based array (RayBiotech Inc.). The specific protein concentration in each kidney lysate was assayed in quadruplicate and quantitated from a standard curve, built into the multiplexed assay, using markers of known concentrations. All diabetic samples $(n=9)$ were positive for HCMV, bacteria or both. All non-diabetic samples $(n=9)$ were negative for HCMV and bacteria. b Tumor-free renal cortical tissues from diabetic and non-diabetic patients were homogenized for DNA, RNA, and protein isolation. RNA integrity and quality were verified (Bioanalyzer, Agilent) before qPCR assay. Shown are the

Non-nephropathic kidneys of diabetic patients are inflamed

Chronic inflammation is a hallmark of diabetes [25]. To investigate effects of inflammation on immunity in diabetes, we assayed adhesion molecules, cytokines, chemokines, and their receptors in renal tissues. Diabetic kidneys had significantly higher levels of proinflammatory molecules (MCP1 , Rantes, TNF- $\alpha$, GRO, and IL-6) compared with controls (Fig. 2a). Transcript levels of IL8, IL18, SELE, ICAM1, $C E B P B$, and $P D G F R A$ were all higher in diabetic kidneys (Fig. 2b).

Inflammation in diabetic kidneys is associated with increased GPCR and MAP kinase activities, and oxidative stress

Signaling pathways associated with chronic inflammation were observed in diabetic kidneys, evidenced by increased results of qPCR array (SABiosciences, Qiagen) that assayed for 21 gene transcript levels using proprietary primers. The transcript level of each gene in each kidney sample was inter-ranked and presented as a pre-assigned color dendogram ( $D$ diabetic patients $1-6, N D$ nondiabetic patients 1-6). Each diabetic sample had evidence of both HCMV and bacterial infection while all non-diabetic samples were negative for both HCMV and bacteria. Yellow-lined borders highlight the only downregulated gene in each category. Fold change (D/ND) for each gene was calculated using the average transcript levels of all subjects in a group, which is accompanied by standard deviation. $(S D)^{*} . P \leq 0.05$, comparison between diabetic and non-diabetic is significant.

protein levels and phosphorylation of PKC- $\beta$ II (Fig. 2c) and NFKB (p65), increased expression of PRKCA, PRKCB, and $P R K C Q$ (Fig. 1b) and increased phosphorylation of MAPK14 (p38 MAPK) and c-Jun N-terminal kinase (JNK). Concomitantly, GPCR signaling molecules that regulate these pathways were induced. GNAQ and $P L C B 1$ were upregulated, as were activators of NFKB (BCL10 and $P T K 2 B$ (PYK2)), p38 MAPK (MAP3K5 or ASK1), and JNK $(P T K 2 B)$ (Fig. 2b). Akt activation in diabetic kidneys was noteworthy because it is known to activate NFkB, JNK, and extracellular signal-regulated kinase (Fig. 2c).

Increased oxidative stress activating NFKB has been implicated in diabetes complications [26]. Diabetic kidneys had increased transcript and membrane-bound protein levels of Nox4, the renal-specific generator of cytosolic reactive oxygen species (ROS) and reduced expression of SOD2, $U C P 2, G P X 3, N Q O 1$, and $C A T$ (Fig. 2b, c). Impaired antioxidative capacity was implied by decreased expression of 

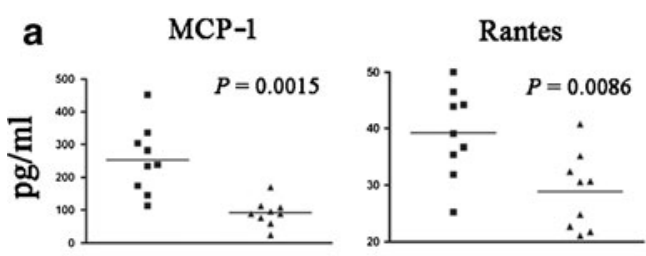

b

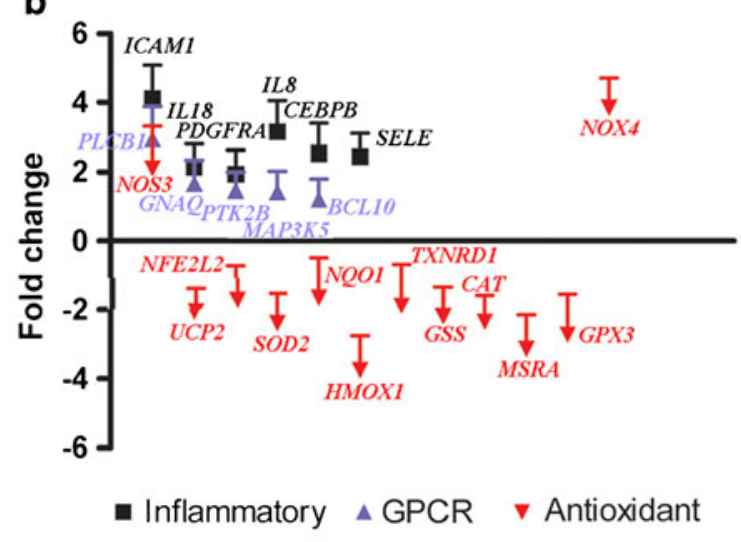

Fig. 2 Diabetic kidneys with clinically normal renal function are inflamed. a Significantly higher concentrations of common proinflammatory cytokines were detected in fresh frozen kidneys of diabetic $(n=$ 9) than in non-diabetic $(n=9)$ subjects using a custom Quantibody ${ }^{\circledR}$ Human Cytokine Array (RayBiotech, Inc.) b Transcript levels of genes implicated in inflammation (6 genes), G protein-coupled receptor $(G P C R)$ signaling (5 genes), and antioxidant pathway (12 genes) were analyzed by qPCR array. Transcripts for genes implicated in inflammatory and GPCR pathways were upregulated in diabetic kidneys while transcripts encoding antioxidant enzymes were downregulated but Nox4, a superior generator, was upregulated. The fold change was calculated from expression levels in fresh frozen kidney tissues of six

NFE2L2, HMOX1, GSS, MSRA, and TXNRD1 (Fig. 2b). In aggregate, these results point to increased oxidative stress in diabetic kidneys.

The paradox of heightened inflammation and impaired immunity in diabetes

The foregoing changes in diabetic kidneys raise a paradox. How do heightened inflammatory responses in diabetes coexist with increased susceptibility to infections, since the former should, in theory, combat microbial invasions more effectively? We hypothesized that the dual effects of TGF- $\beta$ overexpression could help to resolve this paradox (Fig. 1). TGF- $\beta$ potentiates inflammation and induces renal fibrosis but is also important in regulating immune homeostasis [27]. Assaying other immunomodulatory molecules, we showed significant upregulation in diabetic kidneys of IL-4, TSLP, IL-1RN, IL-10, and IL-10Rb, which have antiinflammatory and immunosuppressive actions (Fig. 3a). Conversely, IL1-R1 and IL6-R1 were downregulated in diabetic

\section{c}

PKC- $\beta$ II

P-PKC-BII

p38 MAPK

-p38 MAPK

JNK

P-JNK

Akt

P-Akt

p65

P-p65

Nox4

Sod2

Actin
GRO

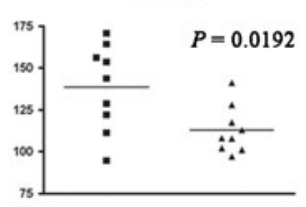

Diabetic
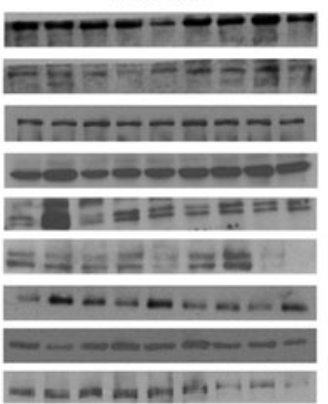

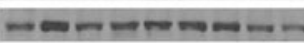

$-\cdots-\cdots-\cdots$

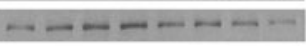

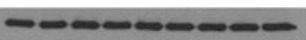

- Diabetic

- Non-diabetic

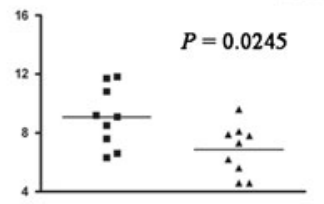

Non-diabetic

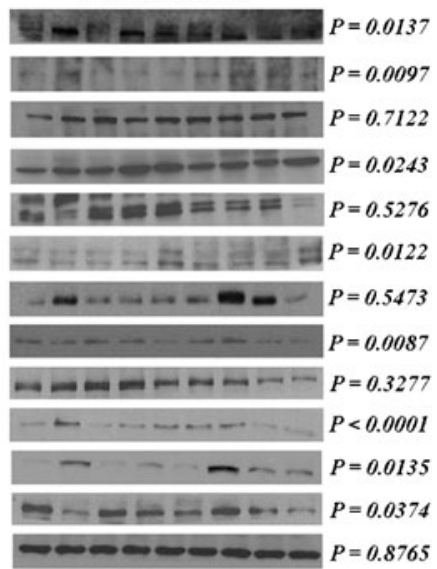

diabetic subjects (each positive for both HCMV and bacteria) compared with an equal number of non-diabetic subjects (negative for both HCMV and bacteria). c Kidney lysates and organelle-specific proteins of diabetic and non-diabetic subjects ( $n=9$ each) were assayed by western blot analysis with the indicated antibodies. All nine diabetic samples were positive for HCMV, bacteria or both. All nine nondiabetic samples were negative for both HCMV and bacteria. The $P$ values compare the expression levels of diabetic and non-diabetic subjects and were calculated based on actin-normalized densitometry values of each group. Western blot experiments for each antibody were repeated at least thrice. Representative blots for each antibody are shown

kidneys. Expression of TNFRI, the major TNF- $\alpha$ receptor, was not significantly altered. Antibacterial chemokines, GCP2 (CXCL6), and MIP-3 $\alpha$ (CCL20), were reduced in diabetic kidneys (Fig. 3a).

Next, we examined negative regulators of inflammatory signaling. In diabetic kidneys, suppression of Toll-like receptor (TLR) signaling was associated with overexpression of its negative regulators (Fig. 3b, c). The level of A20 was higher in diabetic kidneys and correlated with lower transcript and protein levels of TRAF6. Negative regulators of proinflammatory surface receptors were also overexpressed in diabetic kidneys. Triad3A (RNF216) was significantly higher in diabetes and may have contributed to reduced levels of TLR4 protein (Fig. 3c). Similarly, IRAK-M was overexpressed while IRAK4 protein was repressed in diabetic kidneys. Upregulation of transcripts of IL1RL1 (ST2L) and SIGIRR of the TIR superfamily, may also modulate TLR signaling in diabetic tissues (Fig. 3b). Upregulation of ILIRL1 and SIGIRR was consistent with lower protein levels of MyD88, TLR4, IRAK4 and TRAF6 in diabetic 


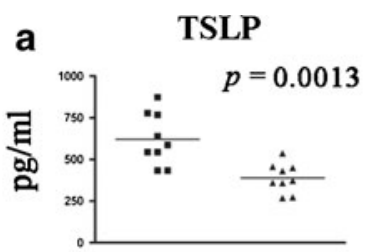

GCP-2
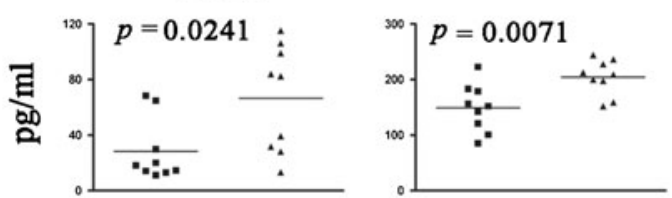

b
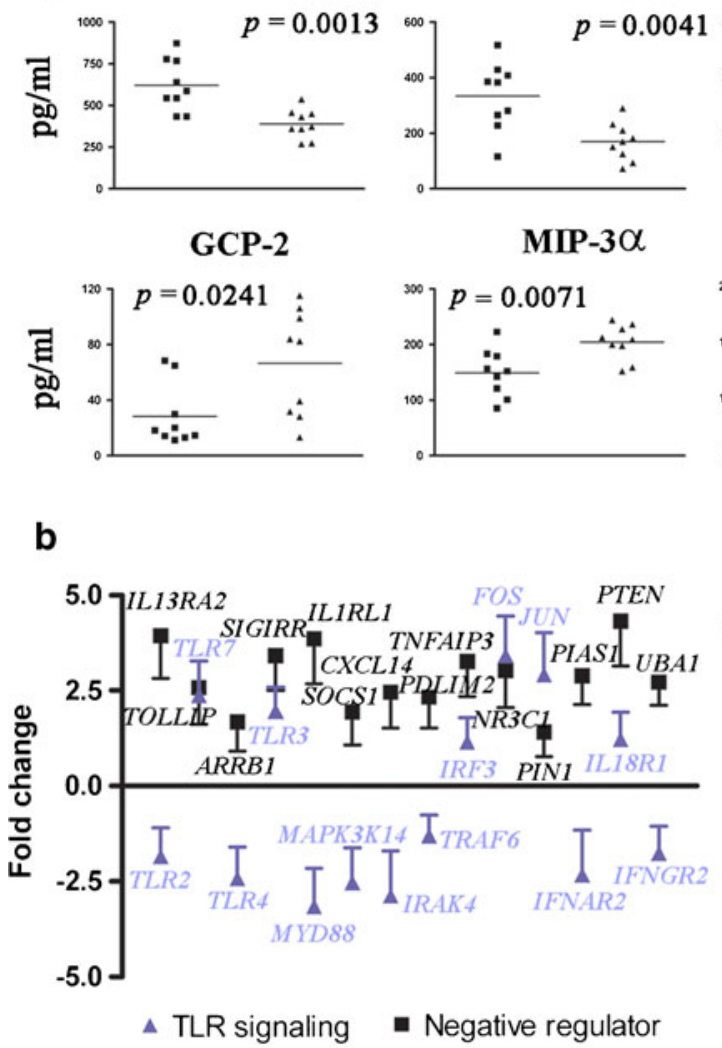

MIP-3 $\alpha$
IL-10Rb

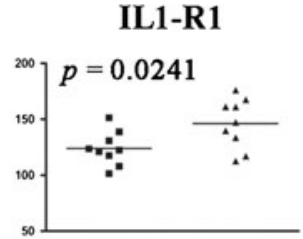

C

A20 Triad3A

IRAK-M

SOCS1

I $\kappa \mathrm{B} \alpha$

TLR4

MyD88

IRAK4

TRAF6

NIK

Actin

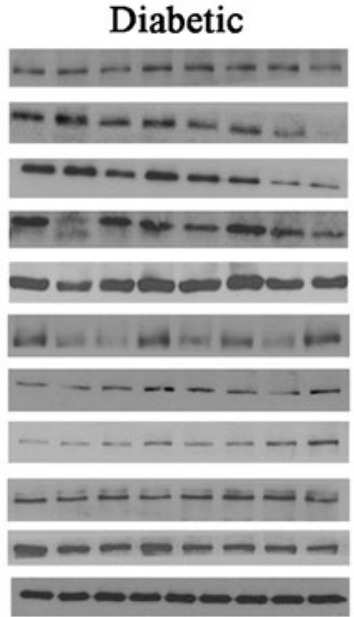

IL-4

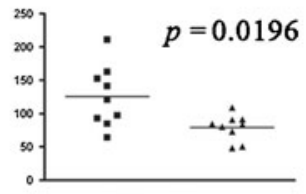

IL-6R

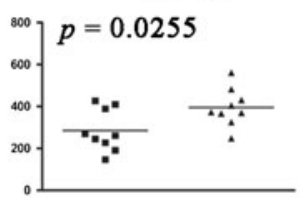

IL-1RN

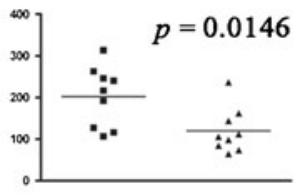

TNF-RI

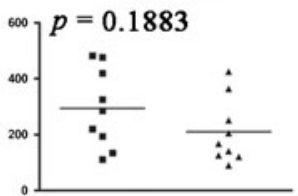

subjects (each positive for both HCMV and bacteria) were compared with an equal number of non-diabetic subjects (negative for both HCMV and bacteria). c Western blot analysis of negative regulators of inflammation (A20, Triad3A, IRAK-M, SOCS1, and $\mathrm{I} \kappa \mathrm{B} \alpha$ ) and TLR signaling molecules (TLR4, MyD88, IRAK4, TRAF6, and NIK) confirmed their higher and lower expression, respectively, in fresh frozen diabetic kidneys. The $P$ value for each paired comparison was calculated from actin-normalized densitometry values of diabetic $(n=8)$ and non-diabetic $(n=8)$ kidneys. All eight diabetic samples were positive for HCMV, bacteria or both. All eight non-diabetic samples were negative for both HCMV and bacteria. Western blot experiments for each antibody were repeated at least thrice. Representative blots for each antibody are shown

Increased expression of negative regulators is associated with impaired immunity and cryptic microbial infections in diabetic kidneys

Transcription of genes encoding antimicrobial peptides (LEAP2, PIGR, and LTF) and complement factors $(C 2$, $C 3, C 4 A$, and $C 5$ ), were all repressed in diabetic kidneys, except for $D E F B 1$, which is known to be glucose-induced (Fig. 4a). Adaptive immunity was also impaired. Genes encoding antigen-presenting proteins, TAP1, TAP2, TAPBP, $H L A-A$, and $H L A-E$, were repressed in diabetic kidneys. High expression of HLA-DPA1 and HLA-DQA1 in diabetic 
a $=$ Antibacterial molecules \& adaptive immunity $\triangle$ Cytosolic pathogen pattern recognition receptor

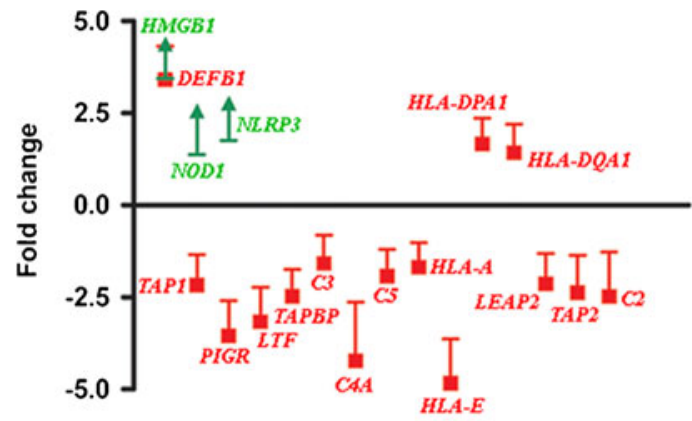

b

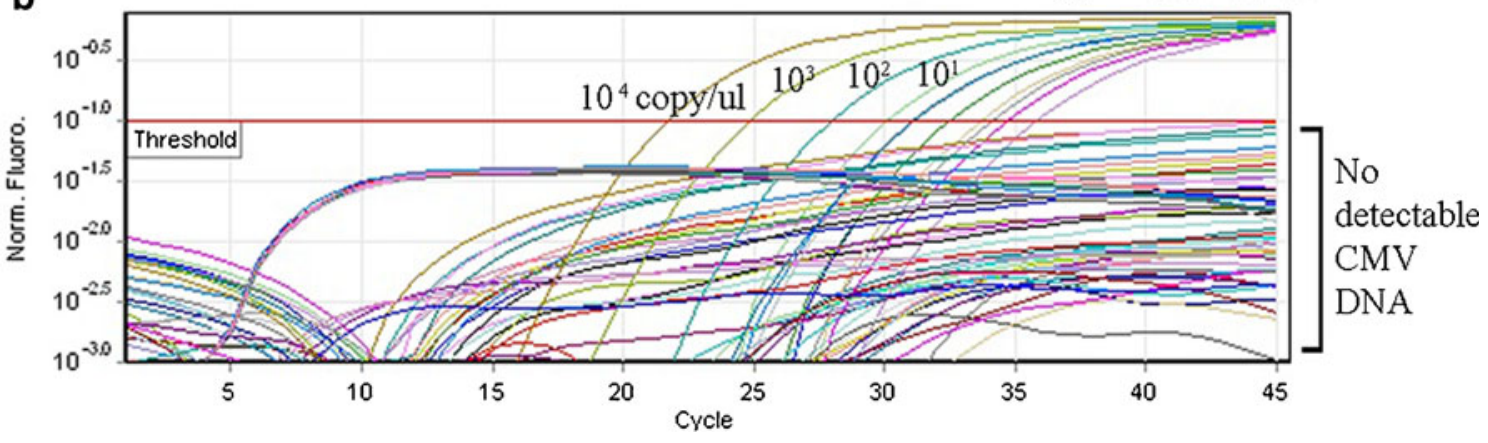

C

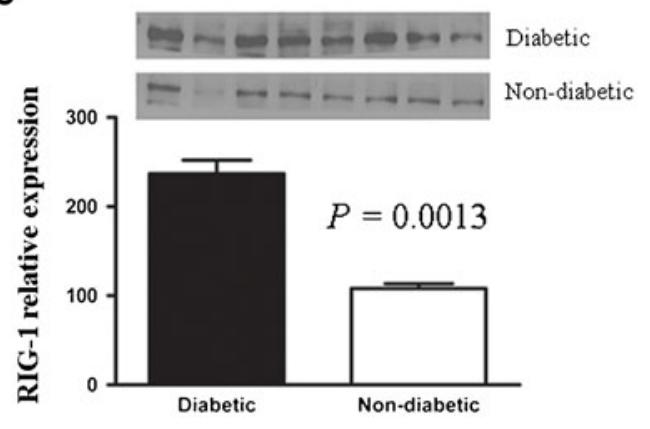

9/16 samples with

$<10$-copy/ul CMV DNA
Fig. 4 Immune-impaired diabetic kidneys had cryptic microbial infections. a Transcript levels of genes encoding antimicrobial peptides, serum complement factors, antigen presentation proteins (15 genes), and cytosolic pathogen pattern recognition receptors (3 genes) were assayed on fresh frozen kidney tissues with qPCR array. The fold change was calculated from renal expression levels of six diabetic subjects (each positive for both HCMV and bacteria) compared with an equal number of non-diabetic subjects (each negative for both HCMV and bacteria). b A clinical diagnostic kit, artus ${ }^{\circledR}$ CMV RG PCR kit (Qiagen $\mathrm{GmbH}$ ), was used to detect HCMV DNA in 16 diabetic (nine fresh frozen and seven FFPE) and 3 non-diabetic kidneys (all fresh frozen). The diagnostic kit detects specific amplification of a 105-bp DNA region of the HCMV genome. Real-time PCR was performed on Rotor-Gene ${ }^{\mathrm{TM}} 3000$ (Roche) as instructed. Data were analyzed with Rotor-Gene ${ }^{\mathrm{TM}}$ software. $\mathbf{c}$ The relative expression levels of RIG-1 were derived from the average of actin-normalized densitometry values of diabetic and non-diabetic kidneys ( $n=8$ each). All eight diabetic samples were positive for HCMV, bacteria or both. All eight non-diabetic samples were negative for both HCMV and bacteria. Western blot analysis was performed at least thrice. A representative blot is shown

TLR3, TLR7, HMGB1, NOD1, and NLRP3) were upregulated in diabetic kidneys positive for HCMV and/or bacterial DNA (Fig. 3b, and 4a, c). Figure 5 shows T and B lymphocyte infiltration in two diabetic kidneys that were positive for HCMV and bacterial DNA.

PKC inhibition and antioxidant reverse impaired immune responses in renal epithelial cells

To identify the resident cell type that plays a major role in the immune responses of whole kidney, we investigated human primary RPTEC and NHMC. RPTEC responded to diabetes-relevant concentrations of metabolic insults (high glucose (HG; $20 \mathrm{mM}$ ), methylglyoxal (MG; $10 \mu \mathrm{M})$, and cytokines (TNF- $\alpha$, IL- 6 , and IL-1 $\beta$ )) not only by activating proinflammatory signaling molecules and increasing expression of proinflammatory cytokines but also by upregulating immunoregulatory molecules (e.g., IL-4, IL-10, TGF- $\beta$, and associated molecular pattern (PAMP) receptors (RIG-1, 
Fig. $5 \mathrm{~T}$ and B lymphocyte infiltrations of diabetic kidneys. Immunohistochemical staining for B (a, c) and T lymphocytes (b, d) of two diabetic kidneys (top case 05-PB20861 and bottom case 06-PB627) with evidence of bacterial and HCMV DNA. Primary antibodies were monoclonal anti-CD20 (B lymphocytes; Dako M0755, clone L26; 1:200 dilution) and polyclonal anti-CD3 (T lymphocytes; Dako A0452; dilution 1:200). The reporter system was EnVision $^{\mathrm{TM}}$ (Dako; horseradish peroxidase and 3,3'diaminobenzidinetetrahydrochloride). Counterstain was hematoxylin. Original magnification was $\times 100$
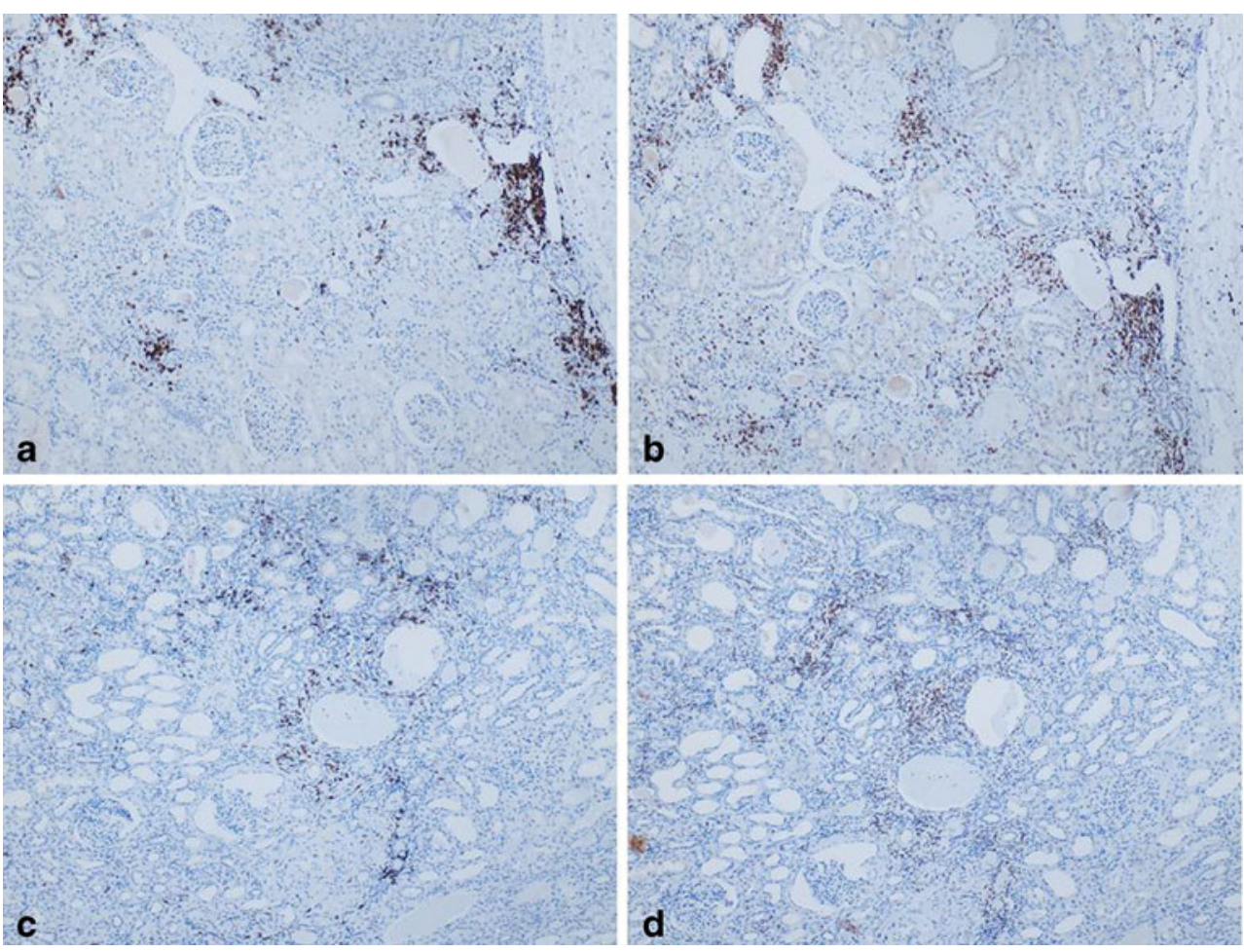

TSLP) and negative regulators of inflammation (e.g., $\operatorname{I\kappa B} \alpha$, IRAK-M, Triad3A, and A20) (Fig. 6). These responses were significant after a 5-day stimulation with any single agent (Fig. 6a, e). In contrast, although mesangial cells exposed to the same agents showed proinflammatory responses, they expressed very low levels of negative regulators and immunoregulatory proteins (Fig. 6a, d). Comparing HG, MG, and cytokines, induction of proinflammatory genes and oxidative stress in both cell types was highest after a 5-day treatment with $\mathrm{MG}$, followed by $\mathrm{HG}$ and cytokines (Fig. 6a). RPTEC had highest expression of negative regulators and lowest expression of TLR signaling molecules in response to MG. However, no significant change was observed in MG-treated NHMC. To determine if the immune response of RPTEC was impaired in conditions that simulated the metabolic milieu of diabetes, we compared protein and mRNA expression profiles of RPTEC challenged with LPS alone (Fig. 6b, c) or a combination of LPS, lipoteichoic acid, and poly(I:C) (Fig. 6d, e), before and after a 5-day incubation with $\mathrm{HG}, \mathrm{MG}$, and cytokines. In the same experiment, we also investigated the effects of DPI, an antioxidant that targets NADPH oxidase, alone or in combination with a PKC inhibitor, Gö6983, on the immune response of RPTEC. RPTEC exposed to conditions mimicking the diabetic metabolic milieu showed a delayed response to LPS stimulation. Activation of TLR leading to NFKB and p38 MAPK activation was evident only $12 \mathrm{~h}$ after LPS stimulation (Fig. 6c) compared with control-unexposed RPTEC that responded to LPS stimulation within $2 \mathrm{~h}$ (Fig. 6b). This delayed response of RPTEC in a diabetic milieu was associated with expression of negative regulators (Fig. 6c) and less robust expression of molecules associated with antimicrobial response (e.g., PIGR, LEAP2, LTF, C2, C3, C4A, C5, TAP1, TAP2, and TAPBP) when challenged with microbial analogs. This was associated with upregulation of multiple immunomodulatory cytokines, negative regulators of inflammation, increased ROS generation, and evidence of impaired intrinsic antioxidant capacity (Fig. 6d, e). The combined presence of Gö6983 and DPI (Fig. 6b, d, e), but not either agent alone (Fig. 6b), mitigated the proinflammatory response, reduced the expression of negative regulators, and restored the prompt immune response of RPTEC challenged with microbial stimulants. Collectively, these in vitro results point to inappropriately heightened counter-regulatory responses to inflammation as a cause of impaired renal epithelial immunity in diabetes and demonstrate reversal by pharmacological interventions directed at PKC activation and ROS generation.

\section{Discussion}

Our results showed that kidneys of diabetic subjects overexpressed inflammatory markers, and had dysregulated signaling pathways involving GPCR, PKC- $\beta$, MAPKs (p38 MAPK and JNK), Akt, TLRs, and NFKB. Our data additionally revealed Nox4-induced ROS generation, hyperglycemia- 
a

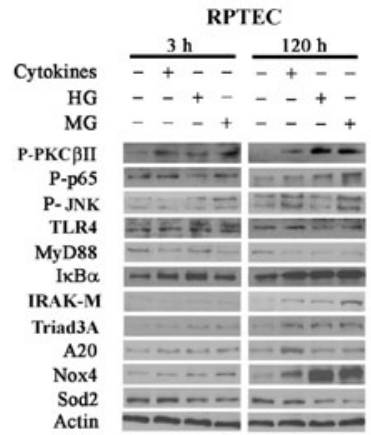

NHMC

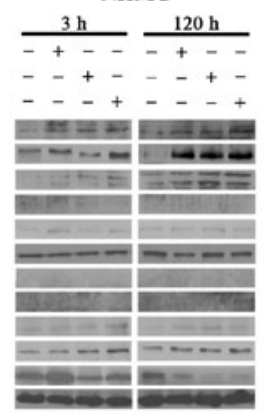

b

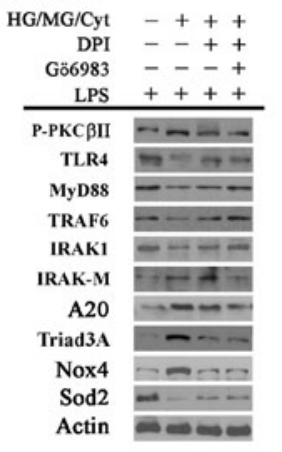

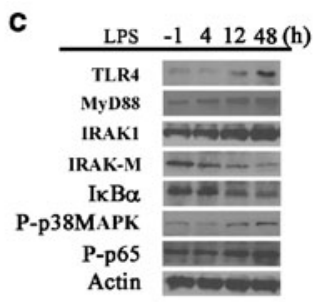

IL-6R

d
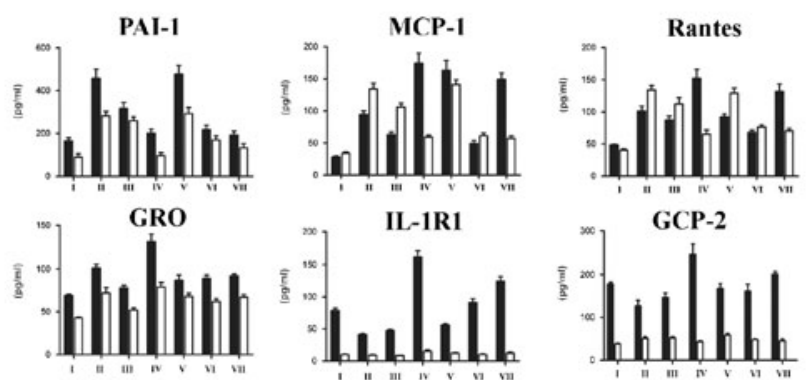

点

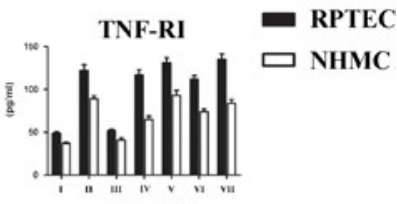

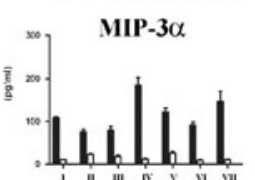
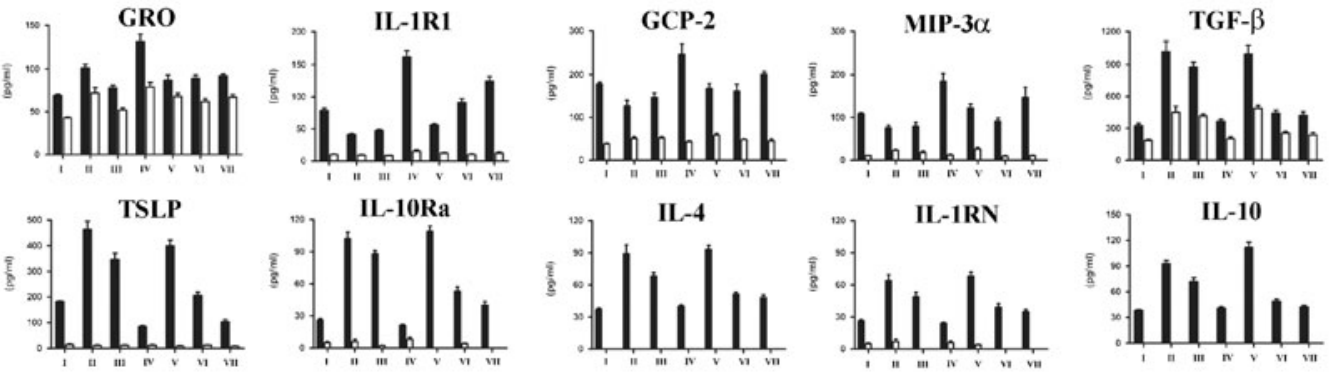

e

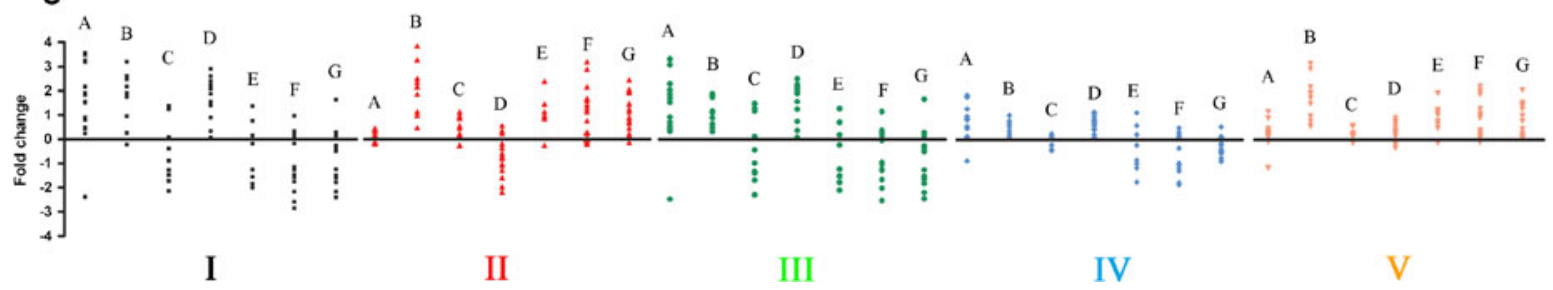

Fig. 6 Renal tubular epithelial cells, but not mesangial cells, modulate overt inflammation via negative feedback mechanisms that impair immunity. a Human renal primary tubular epithelial cells (RPTEC) and mesangial cells (NHMC; Lonza) were cultured with diabetesrelevant concentrations of cytokines (TNF- $\alpha, 50 \mathrm{pg} / \mathrm{ml}$; IL-6, $20 \mathrm{pg}$ / $\mathrm{ml}$; and IL-1 $\beta, 20 \mathrm{pg} / \mathrm{ml})$, high glucose $(H G ; 20 \mathrm{mM})$ or methylglyoxal $(M G ; 10 \mu \mathrm{M})$ for $3 \mathrm{~h}$ or 5 days $(120 \mathrm{~h})$. Protein lysates from each condition $(n=4)$ were prepared for western blot analysis using the indicated antibodies ( $n \geq 3$ for each antibody). b RPTEC were first exposed to a combination of cytokines, high glucose, and methylglyoxal at concentrations stated in (a) for 5 days. The cells were further cultured in fresh medium in the presence of either diphenyleneiodonium chloride $(D P I ; 10 \mu \mathrm{M})$ or combined with Gö6983 $(10 \mathrm{nM})$ for 1 day before challenge with lipopolysaccharide $(L P S ; 1 \mu \mathrm{g} / \mathrm{ml})$ for $2 \mathrm{~h}$. Protein lysates ( $n=4$ for each condition) were analyzed by western blotting ( $\geq 3$ immunoblots for each antibody). c RPTEC cultured in the combined presence of cytokines, $\mathrm{HG}$ and $\mathrm{MG}$ at the aforementioned concentrations for 5 days were challenged with a higher dose of LPS $(10 \mu \mathrm{g} / \mathrm{ml})$ for the indicated time points ( $n=4$ for each time point). Minus $1 \mathrm{~h}$ denotes cells that were not exposed to LPS but were lysed for protein isolation one hour before similarly cultured cells were challenged with LPS. Protein lysates from each condition were prepared for western blotting as described (Figs. 2 and 3 legends). d RPTEC and NHMC were treated as follows before harvesting for protein and total RNA isolations ( $n=4$ for each condition). $I$ Untreated control cells; II incubated with cytokines (TNF- $\alpha, 50 \mathrm{pg} / \mathrm{ml}$; IL-6,
$20 \mathrm{pg} / \mathrm{ml}$; and IL-1 $\beta, 20 \mathrm{pg} / \mathrm{ml}), \mathrm{HG}(20 \mathrm{mM})$, and MG $(10 \mu \mathrm{M})$ for 5 days; $I I I$ incubated with MG $(10 \mu \mathrm{M})$ only for 5 days; $I V$ challenged with LPS $(10 \mu \mathrm{g} / \mathrm{ml})$, lipoteichoic acid $(L T A ; 10 \mu \mathrm{g} / \mathrm{ml})$, and poly(I:C) $(10 \mu \mathrm{g} / \mathrm{ml})$ for $2 \mathrm{~h} ; V$ treated as in (II) before returning to normal culture medium for 1 day and subsequently challenged with microbial stimulants as in (IV); VI cultured as in (II) before treatment with DPI $(10 \mu \mathrm{M})$ and Gö6983 $(10 \mathrm{nM})$ for 1 day in fresh culture medium; VII cultured as in $(V I)$ before challenge as in $(I V)$. Protein lysates were assayed using quantitative protein arrays. e Results of qPCR array plotted as fold change (RPTEC of each condition $c f$. untreated RPTEC). Genes with similar functions or involvement in the same biological process were grouped. $A$ Diabetes related, $B$ inflammatory pathways, $C$ antioxidant system, $D$ negative immune regulators, $E$ interleukin and cytokine receptors, $F$ TLR signaling, and $G$ antimicrobial molecules and adaptive immunity. Roman numerals $(I-V)$ indicate the type of treatment. I RPTEC incubated with cytokines (TNF- $\alpha$, $50 \mathrm{pg} / \mathrm{ml}$; IL-6, $20 \mathrm{pg} / \mathrm{ml}$; and IL- $1 \beta, 20 \mathrm{pg} / \mathrm{ml})$, HG $(20 \mathrm{mM})$, and MG $(10 \mu \mathrm{M})$ for 5 days; II RPTEC challenged with LPS $(10 \mu \mathrm{g} / \mathrm{ml})$, LTA $(10 \mu \mathrm{g} / \mathrm{ml})$ and poly I/C $(10 \mu \mathrm{g} / \mathrm{ml})$ for $2 \mathrm{~h}$; III RPTEC treated as in $(I)$, returned to normal culture medium for 1 day and then challenged with microbial analogs as in $(I I)$; $I V$ RPTEC cultured as in $(I)$ before combined treatment with DPI $(10 \mu \mathrm{M})$ and Gö6983 $(10 \mathrm{nM})$ for 1 day in fresh culture medium; $V$ RPTEC cultured as in $(I V)$ before being challenged as in $(I I)$. The qPCR array experiment for each of the conditions was performed twice 


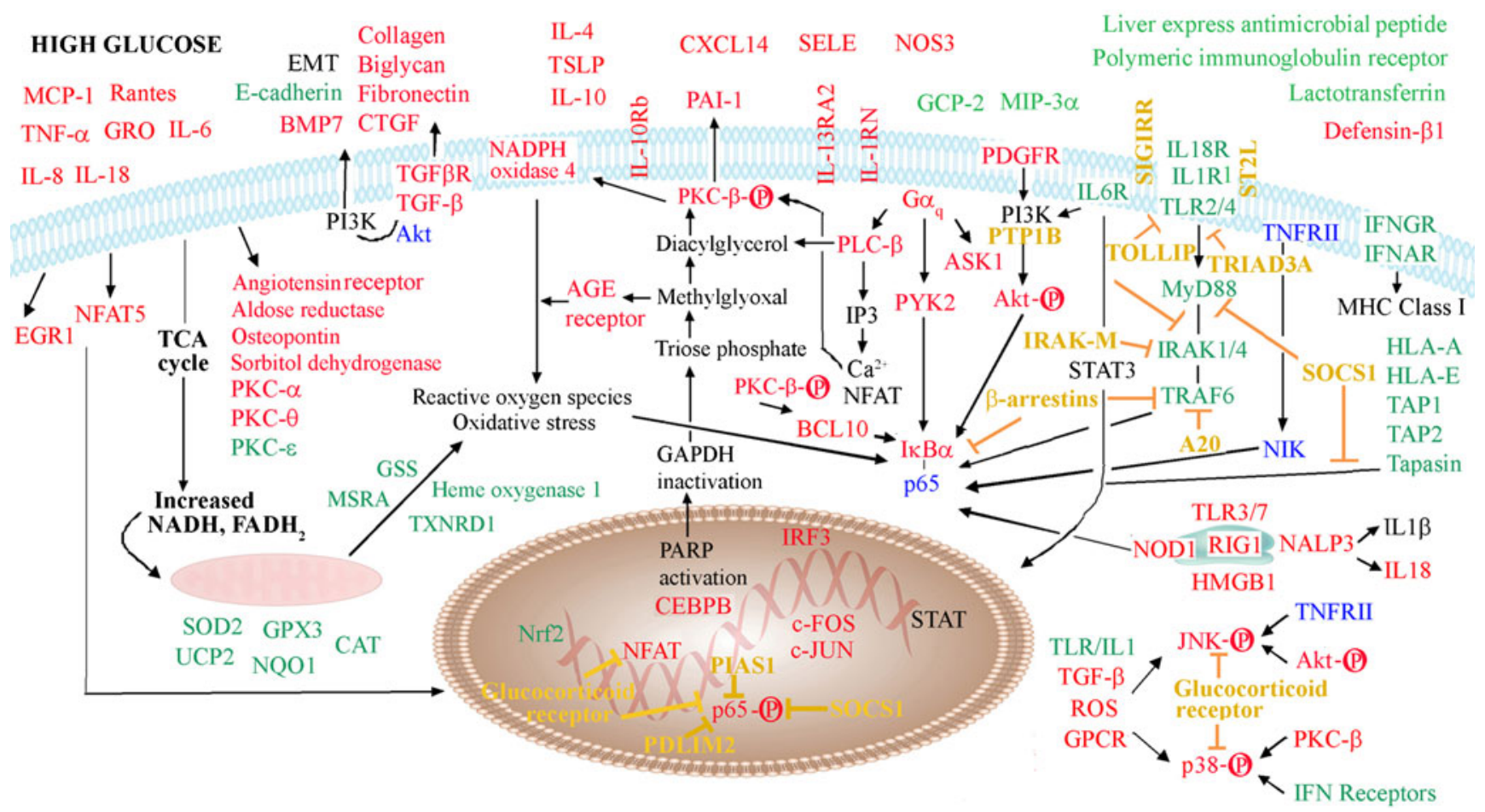

Fig. 7 Proposed model showing molecular mechanisms of dysregulated immune pathways in diabetic renal tissues. Hyperglycemia alters four molecular processes implicated in the pathogenesis of diabetes complications: (1) polyol pathway, (2) increased expression of the receptor for advanced glycation end-product, (3) hexosamine pathway, and (4) protein kinase $\mathrm{C}(P K C)$ signaling. This results in chronic inflammation, accumulation of extracellular matrix proteins, tissue remodeling, mitochondrial dysfunction, and oxidative stress. Chronic activation of the key switches of proinflammatory responses, e.g., PKC- $\beta$, NF-kB, MAPKs, AKT, G protein-coupled receptors (GPCRs), and Toll-like receptors (TLRs) are induced. Oxidative stress from increased generation of reactive oxygen species (ROS), impaired mitochondrial function and diminished antioxidant capacity further exacerbates the proinflammatory state, especially when ROS and PKC- $\beta$ are mutually activating and oxidative stress activates NF- $\mathrm{kB}$. To combat and protect resident cells and tissue from unresolved inflammation, anti-inflammatory mechanisms involving negative regulators of proinflammatory molecules and signaling pathways (yellow letters), immunomodulatory molecules (e.g., IL4, TSLP, and CXCL14), and decoy receptors (e.g., IL1-R1, IL6-R1, and IL13RA2) are chronically activated. These inherent protective mechanisms however compromise antimicrobial defense by interfering with TLR and NF- $\mathrm{kB}$ signaling that in turn reduce expression of key players of innate and adaptive immunity (e.g., circulating antimicrobial peptides and complement factors, and proteins with important roles in antigen presentation). Impaired immunity predisposes to infections that activate cytoplasmic pathogen-associated molecular pattern receptors which recognize internalized bacterial and viral molecules. Information was derived from Ingenuity Pathways Knowledge Base and published literature. Red letters denote upregulation; green letters, downregulation; black letters, mechanisms/modulators not analyzed in this study

endogenous TLR4 ligands, fibronectin, and biglycan. Chronic TLR4 activation leads to hypo-responsiveness to PAMPs [29, 30]. This suggests an explanation for our results showing downregulation of surface TLRs (e.g., TLR2 and TLR4) in diabetic kidneys. In contrast, the expression of cytosolic, endosome-bound TLRs, e.g., TLR3 and TLR7, and an intracellular RNA helicase, RIG-1, were induced in infected diabetic kidneys because they recognize intracellular microbial patterns [31].

Recognizing the inherent heterogeneity of whole tissue analysis, we complemented whole kidney studies with in vitro experiments on subtypes of primary human renal cells. Our results confirmed the active role of renal epithelial cells as key players in renal tissue immunity, in contrast to renal mesangial cells having a more passive function. Although mesangial cells are metabolically vulnerable because they 
cannot restrain intracellular glucose transport and hence tend to overexpress markers of inflammation [32], our data show that their role in tissue immunity is minimal. We propose that renal epithelial cells respond to heightened inflammation and oxidative stress by upregulating immunomodulatory molecules that may influence immune cells of the microenvironment to favor $\mathrm{T}_{\mathrm{H}} 2$ responses. Concurrently, epithelial cells protect themselves from excessive inflammation by expressing a myriad of negative regulators to inhibit inflammatory signaling, particularly the TLR pathway [33]. While it may be effective in helping to extinguish inflammation, this strategy is not without cost to the immune system and the diabetic host, as our findings of cryptic HCMV and/or bacterial infections show. As about one third of diabetic patients develop nephropathy and end-stage renal failure, it is possible that impaired immunity and cryptic infections are contributory factors.

Our in vitro experiments confirmed the role of inflammation and oxidative stress as the main instigators of impaired immunity in diabetes as combined pharmacological intervention with a PKC inhibitor and an antioxidant targeting the ROS generator, NADPH oxidase, but not either agent alone, reversed the molecular dysregulation initiated by common molecular assaults of diabetes and restored a prompt immune response of renal epithelial cells. Our data further confirm and highlight the role of MG in impairing immune functions in diabetes [34]. We show MG to be potent in triggering overexpression of inflammatory molecules and ROS in renal epithelial and mesangial cells, with subsequent impairment of TLR signaling in epithelial cells.

In conclusion, our data suggest an integrated model for diabetes-impaired immunity (Fig. 7). Hyperglycemia induces changes in multiple intrarenal pathways involving mechanisms implicated in tissue damage [32]. These result in mitochondrial dysfunction and oxidative stress, a net effect of diminished antioxidant capability and accumulation of intracellular ROS. Other crucial effects are accumulation of extracellular matrix proteins and proinflammatory responses involving activation of NF-kB, MAPKs, and Akt. Activation of GPCR signaling by ligands such as cytokines, chemokines, lipid and growth factors, and oxidative stress create a vicious cycle of chronic inflammation. In turn, renal epithelial cells mount protective mechanisms to combat these stressors by stimulating the capacity to restore immune homeostasis through inducing $\mathrm{T}_{\mathrm{H}} 2$ and anti-inflammatory responses, and overexpressing negative regulators that intercept proinflammatory responses, especially those directed at TLR signaling and NF-KB. These may interfere with selective transcription of genes regulated by $\mathrm{NFKB}$, other inflammatory transcription factors and the physiological synergy among them [35]. Thus, the expression of target genes of innate and adaptive immunity could be compromised, resulting in increased risk of infections. Internalized bacterial and viral molecules during the course of renal infection may activate cytosolic PAMP receptors, triggering yet another wave of inflammatory response.

Acknowledgments This study is supported by the National Kidney Foundation (NKFRC/2007/04) and the National Cancer Centre, both of Singapore. We thank Hui Sun Leong, Fui Teen Chong, and Su Ghim Sia for assistance in western blot experiments; Betty Yong, Stephen Ma, and Hui Zhen for collating clinical data of study subjects; Evelyn Tan for coordinating provision of fresh frozen human kidney tissues; Athirah Shahab for assistance in processing FFPE kidney tissues; and Han Chong Toh for providing GMP and clinical grade reagents (TNF- $\alpha$, IL-6, and IL$1 \beta)$. We thank Balram Chowbay for material support; Peter Hwang, Paul Macary, and Jinhua Lu for critical reading of the manuscript.

\section{Statement of competing financial interests None}

Open Access This article is distributed under the terms of the Creative Commons Attribution License which permits any use, distribution, and reproduction in any medium, provided the original author(s) and the source are credited.

\section{References}

1. Joshi N, Caputo GM, Weitekamp MR, Karchmer AW (1999) Infections in patients with diabetes mellitus. $\mathrm{N}$ Engl J Med 341:1906-1912

2. Shah BR, Hux JE (2003) Quantifying the risk of infectious diseases for people with diabetes. Diabetes Care 26:510-513

3. Muller LMAJ, Gorter KJ, Hak E, Goudzwaard WL, Schellevis FG, Hoepelman AI, Rutten GE (2005) Increased risk of common infections in patients with type 1 and type 2 diabetes mellitus. Clin Infect Dis 41:281-288

4. The Emerging Risk Factors Collaboration (2011) Diabetes mellitus, fasting glucose, and risk of cause-specific death. N Engl J Med 364:829-841

5. Seacrest AM, Becker DJ, Kelsey SF, LaPorte RE, Orchard TJ (2010) Cause-specific mortality trends in a large populationbased cohort with long-standing childhood-onset type 1 diabetes. Diabetes 59:3216-3222

6. Dubos RJ (1953) Effect of ketone bodies and other metabolites on the survival and multiplication of staphylococci and tubercle bacilli. J Exp Med 98:145-155

7. Geerlings SE, Hoepelman AI (1999) Immune dysfunction in patients with diabetes mellitus. FEMS Immunol Med Microbiol 26:259-265

8. Repine JE, Clawson CC, Goetz FC (1980) Bactericidal function of neutrophils from patients with acute bacterial infections and from diabetes. J Infect Dis 142:869-875

9. Robertson HD, Polk HC Jr (1974) The mechanism of infection in patients with diabetes mellitus: a review of leukocyte malfunction. Surgery 75:123-128

10. Delamaire M, Maugendre D, Moreno M, Le Goff MC, Allannic H, Genetet B (1997) Impaired leucocyte functions in diabetic patients. Diabet Med 14:29-34

11. Summers KL, Marleau AM, Mahon JL, McManus R, Hramiak I, Singh B (2006) Reduced IFN- $\alpha$ secretion by blood dendritic cells in human diabetes. Clin Immunol 121:81-89

12. Andreasen AS, Pedersen-Skovsgaard T, Berg RMG, Svendsen KD, Feldt-Rasmussen B (2010) Type 2 diabetes mellitus is associated with impaired cytokine response and adhesion molecule expression in human endotoxemia. Intensive Care Med 36:1548-1555 
13. Foss NT, Foss-Freitas MC, Ferreira MAN, Cardili RN, Barbosa CMC, Foss MC (2007) Impaired cytokine production by peripheral blood mononuclear cells in type 1 diabetic patients. Diabet Med 33:439-443

14. Plouffe JF, Silva J Jr, Fekety R, Allen JL (1978) Cell-mediated immunity in diabetes mellitus. Infect Immun 21:425-429

15. Mahmoud AA, Rodman HM, Mandel MA, Warren KS (1976) Induced and spontaneous diabetes mellitus and suppression of cell-mediated immunologic responses. Granuloma formation, delayed dermal reactivity and allograft rejection. J Clin Invest 57:362-367

16. Peleg AY, Weerarathna T, McCarthy JS, Davis TM (2007) Common infections in diabetes: pathogenesis, management and relationship to glycaemic control. Diabetes Metab Res Rev 23:3-13

17. Wheat LJ (1980) Infection and diabetes mellitus. Diabetes Care 3:187-197

18. Moutschen MP, Scheen AJ, Lefebvre PJ (1992) Impaired immune responses in diabetes mellitus: analysis of the factors and mechanisms involved. Relevance to the increased susceptibility of diabetic patients to specific infections. Diabet Med 18:187-201

19. McMahon MM, Bistrian RR (1995) Host defenses and susceptibility to infection in patients with diabetes mellitus. Infect Dis Clin North Am 9:1-9

20. Swamy M, Jamora C, Havran W, Hayday A (2010) Epithelial decision makers: in search of the epimmunome. Nat Immunol 11:656-665

21. Dolowschiak T, Chassin C, Ben Mkaddem S, Fuchs TM, Weiss S, Vandewalle A, Hornef MW (2010) Potentiation of epithelial innate host responses by intercellular communication. PLoS Pathog 6: e1001194

22. Kanwar YS, Sun L, Xie P, Liu FY, Chen S (2011) A glimpse of various pathogenic mechanisms of diabetic nephropathy. Annu Rev Pathol 6:395-423

23. Wen L, Ley RE, Volchkov PY, Stranges PB, Avanesyan L, Stonebraker AC, Hu C, Wong FS, Szot GL, Bluestone JA et al (2008) Innate immunity and intestinal microbiota in the development of type 1 diabetes. Nature 455:1109-1113

24. Hills CE, Squires PE (2011) The role of TGF- $\beta$ and epithelial-tomesenchymal transition in diabetic nephropathy. Cytokine Growth Factor Rev 22:131-139

25. Hotamisligil GS (2006) Inflammation and metabolic disorders. Nature 444:860-867

26. Forbes JM, Coughlan MT, Cooper ME (2008) Oxidative stress as a major culprit in kidney disease in diabetes. Diabetes 57:1446-1454

27. Li MO, Wan YY, Sanjabi S, Robertson AL, Flavell RA (2006) Transforming growth factor- $\beta$ regulation of immune responses. Annu Rev Immunol 24:99-146

28. Decker EL (2003) Early growth response proteins (EGR) and nuclear factors of activated T cells (NFAT) form heterodimers and regulate proinflammatory cytokine gene expression. Nucleic Acids Res 31:911-921

29. Okamura Y, Watari M, Jerud ES, Young DW, Ishizaka ST, Rose J, Chow JC, Strauss JF 3rd (2001) The extra domain A of fibronectin activates Toll-like receptor 4. J Biol Chem 276:10229-10233

30. Schaefer L (2005) The matrix component biglycan is proinflammatory and signals through Toll-like receptors 4 and 2 in macrophages. J Clin Invest 115:2223-2233

31. Slater L, Bartlett NW, Haas JJ, Zhu J, Message SD, Walton RP, Sykes A, Dahdaleh S, Clarke DL, Belvisi MG et al (2010) Coordinated role of TLR3, RIG-I and MDA5 in the innate response to rhinovirus in bronchial epithelium. PLoS Pathog 6:e1001178

32. Brownlee M (2005) The pathobiology of diabetic complications. A unifying mechanism. Diabetes 54:1615-1625

33. Akira S, Uematsu S, Takeuchi O (2006) Pathogen recognition and innate immunity. Cell 124:783-801

34. Price C, Knight SC (2009) Methylglyoxal: possible link between hyperglycemia and immune suppression? Trends Endocrinol Metab 20:312-317

35. Smale ST (2010) Selective transcription in response to an inflammatory stimulus. Cell 140:833-844 